\title{
The effect of applying central line insertion and maintenance bundles in a renal dialysis unit - an effort to reduce CRBSI rate
}

\author{
Mohammed Ahmed Fouad ${ }^{1,2}$, Abdelsafi Abbas Gabbad' ${ }^{1}$, Mahmoud AbdelRazik ${ }^{3}$ \\ 1. Department of Public Health, College of Health Sciences at Al-Leith, Umm Al-Qura University, Saudi Arabia. \\ 2 .Microbiology and Immunology Department, Faculty of Medicine, Benha University, Egypt \\ 3. Artificial Kidney Unit, Al-Leath General Hospital.
}

\begin{abstract}
Catheter related blood stream infections (CRBSI) rates were determined before and after the application of central line $(\mathrm{CL})$ insertion and maintenance bundles by means of a prospective surveillance study conducted on patients undergoing regular catheter haemodialysis in the Artificial Kidney Unit of Al-Leith General Hospital, over a period of 12 months. During Phase 1 (baseline period), active surveillance was performed without the implementation of the infection control bundles. CRBSI rates obtained in Phase 1 were compared with CRBSI rates obtained in Phase 2 (intervention period), after implementation of the $C L$ insertion and maintenance bundles.
\end{abstract}

During baseline period 2,854 CL days were recorded, while 2,611 CL days were recorded during the intervention period. The CRBSI rate was 4.9 per 1,000 CL days in baseline period, and in the intervention period the CRBSI rate decreased to 2.3 per $1,000 \mathrm{CL}$ days.

This study shows that the implementation of CVC insertion and maintenance bundles was associated with a significant reduction in the CRBSI rates in the renal dialysis unit of a small hospital. Based on our study, we recommend that these bundles and protocol be adopted in all renal dialysis units.

Keywords: Catheterization, central venous; Infection control and methods; Patient care bundles; Hemodialysis units, hospital

\section{Corresponding Author}

Mohammed Ahmed Fouad

Department of Public Health, College of Health Sciences at Al-Leith, Umm Al-Qura University, Saudi Arabia.

Email: mohmicro@gmail.com 


\section{Introduction}

The survival and life quality of many patients with end stage renal disease (ESRD) are dependent upon the adequacy of dialysis through properly functioning vascular access. ${ }^{1}$ Although central venous catheters (CVCs) are associated with several infections, mechanical and thrombotic complications, ${ }^{2}$ they remain irreplaceable tools of vascular access in the modern dialysis delivery system. ${ }^{3}$

Vascular access-related blood-stream infections and their complications that require hospitalization, account for nearly one third of the cost of ESRD management with reported mortality rates of 12$25.9 \%{ }^{1}$

Central line associated blood stream infections (CLABSIs) are serious but often preventable infections when evidence-based guidelines are followed for the insertion and maintenance of central lines. This preventability is even more acutely apparent in developing countries, where use of these devices may occur in the absence of the most basic infection prevention and control practices and limited availability of supplies. ${ }^{4,5}$

Institute for Health Care Improvement (IHI), USA has developed the concept of 'Bundle' to help clinician deliver bedside care more reliably and effectively. ${ }^{6}$ Care bundles, in general, are groupings of best practices with respect to a disease process that individually improve care, but when applied together result in substantially greater improvement. The $\mathrm{IHI}$ Central Line $(\mathrm{CL})$ Bundle consists of five interventions: hand hygiene; maximal barrier precautions; chlorhexidine skin antisepsis; optimal catheter site selection, with avoidance of the femoral vein for central venous access in adult patients; and daily review of the line necessity, with prompt removal of unnecessary lines. ${ }^{7}$

Most previous studies concentrated mainly on the effect of insertion bundles on the incidence of CLABSI, much less studies addressed the effects of maintenance practices. The renal patient is a chronic patient and a considerable proportion rely on CVCs for dialysis blood access. In comparison with shortterm catheters, in long periods, the value of sound maintenance practices become more prominent.
We hypothesized that the development, implementation, and enforcement of central line insertion and maintenance bundles may result in a decrease in the rate of CLABSI. We therefore developed and implemented two CVC bundles, and evaluated the impact that such an intervention had on reducing the rate of CLABSI per 1,000 line days during the 6 months before and after implementation of the policy.

\section{Subjects and Methods}

\section{Setting and study design}

This study was conducted on patients undergoing regular catheter haemodialysis in the Dialysis Unit of Al-Leith General Hospital at Al-Leith, Saudi Arabia, over a period of 12 months between April 2013 and March 2014.

This is a prospective pre- and post-intervention study. It was performed in two phases: Phase 1 (baseline period) and Phase 2 (intervention period). The following data were collected in the six months prior to and the six months following the start of the policy: (1) number of central line days; (2) number of central venous catheter associated blood stream infections; (3) central venous catheter associated bloodstream infections per 1000 central line days; and (4) central line bundle compliance;

\section{Definitions}

A device day was defined by a patient having a single CVC for a whole or part $24 \mathrm{~h}$ period; two catheters for a part or whole $24 \mathrm{~h}$ period was defined as two device days and so on.

CRBSI is defined according to CDC as bacteraemia/ fungaemia in a patient with an intravascular catheter with at least 1 positive blood culture obtained from a peripheral vein, clinical manifestations of infections (i.e., fever, chills, and/or hypotension), and no apparent source for the BSI except the catheter. One of the following should be present: a positive semiquantitative (15 CFU/catheter segment) or quantitative $\left(10^{3} \mathrm{CFU} /\right.$ catheter segment catheter) culture whereby the same organism (species and antibiogram) is isolated from the catheter segment and peripheral blood; simultaneous quantitative blood cultures with a 5:1 ratio CVC versus peripheral; differential period of 
CVC culture versus peripheral blood culture positivity of 2 hours. ${ }^{8}$

\section{Diagnosing CRBSI and microbial identification}

CRBSI was diagnosed by a differential time to positivity of CVC culture versus peripheral blood culture of 2 hours. Microbial identification was done by Gram stained film, colony characteristics, and biochemical reactions. API 20E was used to confirm the identity of Gram negative isolates. Gram positive isolates were identified by culture characteristics, catalase and coagulase tests.

\section{The Intervention}

We developed two central line infection control bundles derived from published strategies: the central line insertion bundle and the central line maintenance bundle. Each bundle consists of five evidence-based procedures. Our intervention consists of implementing the components of these two bundles.

The central line insertion bundle elements were: (1) hand hygiene before insertion; (2) maximal barrier precautions (large sterile drape, sterile gloves, sterile gown, cap, and mask); (3) skin antisepsis with $2 \%$ chlorhexidine in $70 \%$ alcohol; (4) optimal catheter site selection (the femoral site should be avoided); (5) application of sterile gauze or transparent dressing over catheter exit site.

The maintenance bundle elements were: (1) hand hygiene before and after all line maintenance/access procedures; (2) skin antisepsis with chlorhexidine in alcohol during dressing changes; (3) regular replacement of dressing or if it becomes damp, loosened, or visibly soiled; (4) daily review of line necessity, with prompt removal of unnecessary lines; (5) following hub decontamination procedure before each hub access. The access hub is rubbed for at least 15 seconds using an antiseptic containing 70\% isopropyl alcohol ('scrub the hub').

The process of implementing these bundles involved (1) staff education sessions using PowerPoint presentations; (2) the distribution of a CVC Bundle handbook that explain the bundle elements to all staff; (3) some Posters were put in the work area that motivate staff to follow the bundle elements to save lives; (4) creating catheter insertion pack and maintenance pack; (5) feedback of surveillance data.

The compliance rates for both bundles were measured in phase 2 using checklists which were filled by the nurse during or after the procedure. Compliance with a bundle was defined as completion of all elements of the bundle.

\section{Statistical methods}

Patient characteristics during the baseline and intervention periods were compared using Fisher's exact test for dichotomous variables and unmatched Student's t-test for continuous variables. Relative risk (RR) ratios with 95\% confidence intervals (Cls) were calculated using SPSS for comparisons of rates of CRBSI at baseline and the subsequent intervention period. P-values $<0.05$ by two-sided tests were considered to be significant.

\section{Results}

Over the whole 12 months study period from April 2013 to March 2014, a total of 22 patients were enrolled with $5465 \mathrm{CL}$ days.

Regarding CRBSI rates, during Phase 1 (baseline period), there were 2,854 documented CL days. There were 14 CRBSIs, for an overall baseline rate of 4.9 CRBSIs per 1,000 CL days. In Phase 2, after the implementation of the two infection control bundles program, there were 2,611 CL days. There were 6 CRBSIs, for an overall rate of 2.3 CRBSIs per 1,000 $\mathrm{CL}$ days. These results showed a $53.2 \%$ reduction of CRBSI rate from the baseline (RR 3.19, 95\% Cl 1.059.68, $\mathrm{P}=0.03$ ) (Table I). The monthly CRBSI rates are shown in Fig 1. Zero infection rate was observed in the $4^{\text {th }}$ month after intervention.

The compliance rates were measured over the six months intervention period, $22 \mathrm{CL}$ insertion and $1110 \mathrm{CL}$ maintenance checklists were filled. For the insertion bundle, the monthly compliance rate ranged from $50 \%$ to $100 \%$ and the overall compliance level was $81.8 \%$ over the whole intervention period. For the maintenance bundle, the monthly compliance rate ranged from $84 \%$ to $97 \%$ and the overall compliance level was $92.9 \%$ over whole the intervention period. 
Table I. CRSBI rate in phase 1 (base line period) and in phase 2 (intervention period)

\begin{tabular}{|c|c|c|c|c|}
\hline & Baseline period & Intervention period & $\operatorname{RR}(95 \% \mathrm{Cl})$ & $P$ value \\
\hline No. of CRBSI & 14 & 4 & \multirow{3}{*}{$3.19(1.05-9.68)$} & \multirow{3}{*}{0.03} \\
\hline No. of CL days & 2854 & 2611 & & \\
\hline CRBSI rate per $1,000 \mathrm{CL}$ days & 4.91 & 2.3 & & \\
\hline
\end{tabular}

RR: relative risk; CRBSI: catheter related blood stream infection; $\mathrm{CL}$ : central line; $\mathrm{Cl}$ : confidence interval

The microorganisms profile is shown in Table II. In this study the most common microorganisms isolated from CRBSI infection cases were Staphylococcus aureus (33\%), coagulase negative Staphylococcus spp. (17\%), Pseudomonas aeruginosa (11\%), Escherichia coli (11\%), and Acinetobacter spp. (11\%). Other organisms accounted for $(18 \%)$.

\section{Discussion}

End stage renal disease patients are dependent on their permanent vascular access to receive life-sustaining dialysis. Unfortunately, vascular access is associated with many complications that significantly increase mortality and morbidity among this population. Central Venous catheters (CVCs) remain indicated in many renal dialysis patients either temporarily or permanently. CVCs however are associated with relatively higher infection risk than other methods of vascular access. ${ }^{9,10}$ Therefore, many efforts had been exerted to try to reduce $\mathrm{CVC}$ related infection risk, and one apparently successful approach was the 'Bundle' approach.

Although bundle of care approach was studied extensively in ICUs ${ }^{7,11-13}$ and paediatric wards, ${ }^{14,15}$ no studies were conducted in renal dialysis patients. The objective of this study was to apply the infection control bundle approach to renal haemodialysis patients to estimate its effectiveness in reducing CRBSI rate. The baseline CRBSI rate observed was 4.9/1,000 catheter days which is relatively high when compared to other reports from other developed countries, for

Table II. Isolated Microorganisms form CRBSI cases in the two stages of the study

\begin{tabular}{lrrr} 
Microorganism & $\begin{array}{r}\text { Baseline } \\
\mathbf{\%}(\mathbf{n})\end{array}$ & $\begin{array}{r}\text { Intervention } \\
\mathbf{\%}(\mathbf{n})\end{array}$ & $\begin{array}{r}\text { Total } \\
\mathbf{\%}(\mathbf{n})\end{array}$ \\
\hline Staphylococcus aureus & $29(4)$ & $50(2)$ & $33(6)$ \\
\hline Coagulase Negative Staphylococcus spp. & $14(2)$ & $25(1)$ & $17(3)$ \\
\hline Enterococcus faecalis & $7(1)$ & $0(0)$ & $6(1)$ \\
\hline Pseudomonas aeruginosa & $14(2)$ & $0(0)$ & $11(2)$ \\
\hline Escherichia coli & $7(1)$ & $25(1)$ & $11(2)$ \\
\hline Acinetobacter spp. & $14(2)$ & $0(0)$ & $11(2)$ \\
\hline Enterobacter cloacae & $7(1)$ & $0(0)$ & $6(1)$ \\
\hline Klebsiella pneumoniae & $7(1)$ & $0(0)$ & $6(1)$ \\
\hline Total & $\mathbf{1 0 0}(\mathbf{1 4 )}$ & $\mathbf{1 0 0 ( 4 )}$ & $\mathbf{1 0 0}(\mathbf{1 8})$ \\
\hline
\end{tabular}

CRBSI: catheter related blood stream infection 


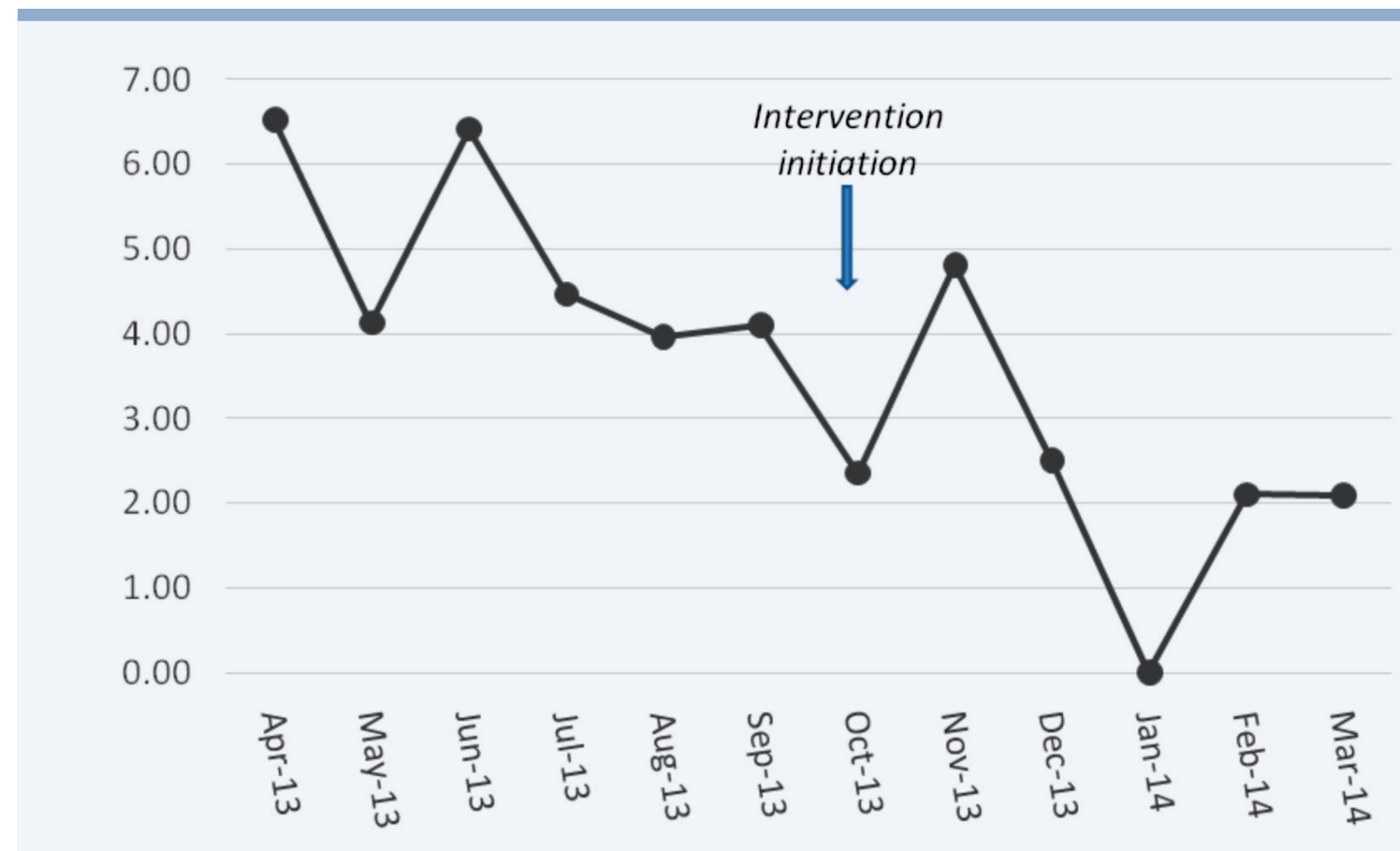

Figure 1. CRBSI rate per 1,000 catheter days over the study period

example 0.85/1,000 CVC-days was reported in north America $^{16}$ and 1.46/1,000 CVC-days was reported in Australia. ${ }^{17}$

This study achieved a 52\% reduction in CRBSI rate. Although no previous studies applied the bundle approach to the haemodialysis patients, one study applied 2 elements found in our insertion bundle which were the use of $2 \%$ chlorhexidine with $70 \%$ alcohol swab sticks for exit-site care and the use of $70 \%$ alcohol pads to perform "scrub the hubs" in dialysis-related CVC care procedures. That study achieved around $22 \%$ reduction in BSI rates. ${ }^{16}$

In this study the high reduction in CRBSI rate may be partially attributable to the relatively high baseline CRBSI rate which may reflect some malfunctioning in the existing infection control program in the facility. Similar high reduction rates were achievable in other studies when the pre-existing basal infection rate was high. ${ }^{12}$ The high reduction in infection rate may be also attributable to the simultaneous application of two bundles, in other study the simultaneous application of CVC insertion and maintenance bundles in a paediatric intensive care unit resulted in an astonishing $70 \%$ reduction in central line-associated bloodstream infection rate from 7.8 infections per
1000 catheter-days to 2.3 infections per 1000 catheterdays. ${ }^{15}$

Many studied concentrated on one bundle alone, usually the insertion bundle, ${ }^{13}$ but in renal dialysis patients, the maintenance gains more importance due to the chronic nature of the disease and prolonged catheter use period.

During this study, a relatively high level of compliance was achieved, the compliance level was better for Maintenance bundle $(92.88 \%)$ than the insertion bundle (81.82\%).

Gram positive cocci were the most common organisms isolated from CRSBI cases (56\%). In another study, Gram positive cocci accounted for $52 \%$ of vascular access-related infections. ${ }^{18}$ The most common isolated organism was Staphylococcus aureus, which agrees with the results of another study, performed in the same country which Staphylococcus aureus was responsible for $29 \%$ of cases among haemodialysis patients. ${ }^{19}$

In summary, this study showed that the application of $\mathrm{CL}$ insertion and Maintenance bundles was successful in reducing CRSBI rates among renal dialysis patients. 


\section{References}

1. Saxena AK, Panhotra BR. Haemodialysis catheter-related bloodstream infections: current treatment options and strategies for prevention. Swiss Medical Weekly 2005; 135(910): $127-138$

2. Mermel LA. Prevention of intravascular catheter-related infections. Annals of Internal Medicine 2000; 132(5): 391-402. http://dx.doi.org/10.7326/0003-4819-132-5-20000307000009

3. Di Benedetto A, Basci A, Cesare S, Marcelli D, Ponce P, Richards N. Increased use of catheters as vascular access: is it justified by patients' clinical conditions? The Journal of Vascular Access 2007; 8(1): 21-27.

4. Zaidi AK, Huskins WC, Thaver D, Bhutta ZA, Abbas Z, Goldmann DA. Hospital-acquired neonatal infections in developing countries. Lancet 2005; 365(9465): 1175-1188. http://dx.doi.org/10.1016/S0140-6736(05)71881-X

5. Rosenthal VD. Central line-associated bloodstream infections in limited-resource countries: a review of the literature. Clinical Infectious Diseases 2009; 49(12): 1899-1907. http:// dx.doi.org/10.1086/648439

6. Berwick DM, Calkins DR, McCannon CJ, Hackbarth AD. The 100,000 lives campaign: setting a goal and a deadline for improving health care quality. JAMA 2006; 295(3): 324-327. http://dx.doi.org/10.1001/jama.295.3.324

7. Furuya EY, Dick A, Perencevich EN, Pogorzelska M, Goldmann D, Stone PW. Central line bundle implementation in US intensive care units and impact on bloodstream infections. PloS One 2011; 6(1): e15452. http://dx.doi.org/10.1371/ journal.pone.0015452

8. Horan TC, Andrus M, Dudeck MA. CDC/NHSN surveillance definition of health care-associated infection and criteria for specific types of infections in the acute care setting. American Journal of Infection Control 2008; 36(5): 309-332. http:// dx.doi.org/10.1016/j.ajic.2008.03.002

9. Rosenbaum D, MacRae JM, Djurdjev O, Levin A, Werb R, Kiaii M. Surveillance cultures of tunneled cuffed catheter exit sites in chronic hemodialysis patients are of no benefit. Hemodialysis International Symposium on Home Hemodialysis 2006; 10(4): 365-370. http://dx.doi.org/10.1111/j.15424758.2006.00131x
10. Ishani A, Collins AJ, Herzog CA, Foley RN. Septicemia, access and cardiovascular disease in dialysis patients: the USRDS Wave 2 study. Kidney International 2005; 68(1): 311-318. http://dx.doi.org/10.1111/j.1523-1755.2005.00414.x

11. Longmate AG, Ellis KS, Boyle $L$, et al. Elimination of centralvenous-catheter-related bloodstream infections from the intensive care unit. BMJ Quality \& Safety 2011; 20(2): 174180. http://dx.doi.org/10.1136/bmjqs.2009.037200

12. Galpern D, Guerrero A, Tu A, Fahoum B, Wise L. Effectiveness of a central line bundle campaign on line-associated infections in the intensive care unit. Surgery 2008; 144(4): 492-495. http://dx.doi.org/10.1016/j.surg.2008.06.004

13. McLaws ML, Burrell AR. Zero risk for central line-associated bloodstream infection: are we there yet? Critical Care Medicine 2012; 40(2): 388-393. http://dx.doi.org/10.1097/ CCM.0b013e318232e4f3

14. Rinke ML, Chen AR, Bundy DG, et al. Implementation of a central line maintenance care bundle in hospitalized pediatric oncology patients. Pediatrics 2012; 130(4): e996-e1004. http://dx.doi.org/10.1542/peds.2012-0295

15. Costello JM, Morrow DF, Graham DA, Potter-Bynoe G, Sandora TJ, Laussen PC. Systematic intervention to reduce central lineassociated bloodstream infection rates in a pediatric cardiac intensive care unit. Pediatrics 2008; 121(5): 915-923. http:// dx.doi.org/10.1542/peds.2007-1577

16. Rosenblum A, Wang W, Ball LK, Latham C, Maddux FW, Lacson Jr E. Hemodialysis Catheter Care Strategies: A ClusterRandomized Quality Improvement Initiative. American Journal of Kidney Diseases 2014; 63(2): 259-267. http:// dx.doi.org/10.1053/j.ajkd.2013.08.019

17. Chu G, Adams K, Crawford S. Improving catheter-related blood stream infection in haemodialysis patients using a practice development framework. Renal Society of Australasia Journal 2013; 9(1): 16-21.

18. Saad TF. Bacteremia associated with tunneled, cuffed hemodialysis catheters. American Journal of Kidney Diseases 1999; 34(6): 1114-1124. http://dx.doi.org/10.1016/S02726386(99)70018-1

19. Saxena AK, Panhotra BR, Naguib M, et al. Vascular access related septicemia in hemodialysis: a focus on bacterial flora and antibiotic access salvage. Saudi Journal of Kidney Diseases and Transplantation 2002; 13(1): 29-34 